\title{
De la consultation à l'ordonnance. Gaspar de Pons et la réforme des institutions financières de la Monarchie hispanique (1590-1593)
}

\author{
Sylvain André
}

\begin{abstract}
Sylvain André : Sylvain André est actuellement doctorant contractuel du CAER (Centre Aixois d'Études Romanes, CAER (EA 854)) à l'Aix-Marseille Université (AMU) / École des hautes études hispaniques et ibériques (Casa de Velázquez). Son projet de doctorat porte sur la méthode gouvernentale employée par Philippe II à la fin de son règne. Plus particulièrement, il s'agit d'analyser l'implication d'une institution centrale créée à la fin des années 1580, connue sous le nom de Junta de Noche ou Junta de Gobierno qui joua un rôle prépondérant dans le processus de rationalisation du gouvernement.
\end{abstract}

\section{DOI: $10.25518 / 1370-2262.421$}

\section{Résumé :}

Cet article se propose d'étudier les coulisses de la réforme de l'administration financière qui eut lieu en novembre 1593, à la fin du règne de Philippe II. En suivant la trajectoire politique de son maître d'œuvre et plus fervent défenseur, Gaspar de Pons, nous souhaitons montrer sous un jour nouveau les enjeux politiques liés à l'édiction de ces ordonnances. A travers les multiples commissions (Juntas) convoquées et les consultations qui jalonnent cet épisode de l'histoire de l'administration espagnole, nous mettrons également en lumière les pratiques gouvernementales de l'époque. Souvent pensée comme le lieu privilégié d'affrontement entre juristes (letrados) et noblesse de conseil, la cour de Philippe II permit également à d'autres types de personnages d'évoluer dans les cercles de décision, lesquels, sans mettre à l'écart les juristes, favorisèrent une autre façon de penser l'action de gouvernement, davantage fondée sur la collaboration et le recours à des expertises nouvelles.

Mots-clés : Gaspar de Pons - Administration financière - Réforme - Monarchie hispanique

\section{Introduction}

Les ordonnances publiées par Philippe II le 20 novembre 1593 au Pardo avaient pour finalité de définir et d'agencer les fonctions et le fonctionnement des institutions centrales des finances, à savoir : le Consejo de Hacienda, la Contaduría Mayor de Hacienda et la Contaduría Mayor de Cuentas1. Jusqu'alors, ces instances ne s'étaient forgées que par agrégation de prérogatives et de compétences mais n'avaient jamais fait l'objet d'une réflexion d'ensemble de la part de Charles Quint ni de Philippe II. Aussi, de l'incertitude quant aux attributions de chaque organe découlaient quotidiennement des incompréhensions, des pertes de temps et des conflits juridictionnels toujours plus récurrents qui minaient l'action du gouvernement. Pire encore : cette situation rendait particulièrement difficile le contrôle des ministres à la cour (conseillers, secrétaires) ainsi que des officiers royaux mandatés dans les divers territoires de la monarchie. Ce constat était exposé dès les premières lignes des ordonnances: "Sachez que nous avons été informés que, du fait de n'avoir pas donné d'ordre particulier sur les procédures de bureaux devant être observées dans de nombreuses affaires traitées au Consejo de hacienda, tant pour ce qui touche au gouvernement, 
De la consultation à l'ordonnance. Gaspar de Pons et la réforme des instituti...

bénéfice et juste perception des finances que pour ce qui a trait à l'administration de la justice et à sa juste et brève exécution, il s'en est ensuivi toutes sortes de doutes et de différends qui retardent tout au détriment des parties. Désireux de résoudre ces problèmes et d'ordonner à ce sujet ce qu'il convient... »2. C'était, à notre connaissance, la première tentative d'approche globale de la question. En effet, bien loin de montrer une quelconque préoccupation pour la structuration des instances gouvernementales, les hommes de l'entourage du roi s'évertuaient alors à résoudre la difficile situation financière de la monarchie par la recherche de moyens ponctuels afin de subvenir aux besoins financiers de la couronne. Cependant, par l'influence d'autres personnages, déliés de toute fonction au sein du système conciliaire et conviés au début des années 1590 à participer à de multiples commissions créées afin de renflouer le trésor, l'idée de réformer l'administration des finances émergea peu à peu.

Pour comprendre cet épisode de l'histoire de l'administration de la Monarchie hispanique, il convient de prendre en compte plusieurs paramètres. Il faut en premier lieu considérer que la dernière décennie du règne de Philippe II fut marquée par la recherche constante de nouveaux expédients financiers (arbitrios ou medios) visant à acquérir le plus rapidement possible des liquidités. Qu'elles aient été la résultante de choix fiscaux (taxations commerciales, négociations de services avec les Cortès, établissement de taux de vente fixes de censos et de juros, etc.) ou de stratégies économiques au sens large (limitation du pouvoir des banquiers, vente de juridictions de la couronne, etc.), ces solutions avaient en commun d'être, dans la majorité des cas, des réponses urgentes à une situation financière délicate $\underline{3}$.

Ensuite, il faut rappeler qu'à cette même époque commença de se développer un genre nouveau d'écrits politiques, rassemblés sous le terme arbitrismo, dont la vocation était de détecter les problèmes financiers de la monarchie et d'y proposer des solutions sous la forme de requêtes (memoriales) adressées au roiı․ Ces textes, dont les premiers exemples remontent à la moitié du $\mathrm{XVI}^{\mathrm{e}}$ siècle, tendirent à la toute fin du règne à se multiplier, avant de connaître leur apogée au XVII siècle en devenant les vecteurs de dénonciation de ce qui était alors perçu comme une forme de décadence sous les Habsbourg․․ Dans une certaine mesure, leurs auteurs, les arbitristas, peuvent être considérés comme les premiers théoriciens ayant cherché à appréhender le politique à travers la question économique et, par conséquent, par le biais de considérations monétaires, agraires, commerciales, militaires, démographiques, etc. Bien entendu, le bien de la res publica et des royaumes, la défense du catholicisme, la conservation de la monarchie étaient invoqués dans ces discours, mais on peut se demander s'il ne s'agissait pas tout simplement de figures imposées afin de faciliter par ailleurs le développement d'autres types d'analyses que celles dont se nourrissait la littérature politique du temps, la tratadística.

A la croisée de la réflexion théorique et de l'activité gouvernementale, certains hommes parvinrent néanmoins à se frayer un champ d'action en suscitant l'intérêt du monarque grâce à leurs propositions (à court et moyen terme à la fois), en s'insérant dans les rouages de l'appareil de gouvernement (relation avec les secrétaires, les conseillers) et en acquérant certaines méthodes de travail (participation à des commissions d'experts ou Juntas).

Un homme en particulier incarnait alors cette position à la cour : Gaspar de Pons, dont la trajectoire est assez atypique et demeure largement méconnue $\underline{6}$. Pons est passé à la postérité comme arbitrista.

Mais bien plus que cela, il fut l'un des rares arbitristas de la fin du XVI ${ }^{\mathrm{e}}$ siècle à accéder aux plus hauts cercles de décision. Maniant tour à tour la plume - rédaction de requêtes, de propositions, etc. - et l'art oratoire - intervention dans de multiples commissions spécialisées -, Pons accéda 
finalement au centre névralgique du pouvoir. En étudiant sa participation à la politique du temps brouillons, correspondances, billets, comptes rendus de réunions -, nous voulons montrer que le processus d'élaboration des ordonnances de novembre 1593, sous sa houlette, s'enracina dans des pratiques gouvernementales favorisant la collaboration de personnages aux domaines d'expertise et aux expériences variés. En s'entourant de divers secrétaires, de membres de grands Conseils mais aussi d'officiers subalternes, de prélats ou encore d'arbitristas et de penseurs politiques (tratadistas), et ayant su gagner la confiance du monarque, Pons parvint à imposer un certain nombre de ses idées et conduisit lui-même l'élaboration de la réforme. A travers ce personnage, à la fois penseur et homme de pouvoir, nous découvrirons, d'une part, les enjeux et le contenu de la réforme de 1593 et, d'autre part, les coulisses du gouvernement.

Pour commencer, nous étudierons les voies par lesquelles les idées de Pons circulèrent à la cour parmi les secrétaires, les membres et les présidents de Conseils. Dans le même temps, nous essaierons de montrer comment les positions qu'il défendait au sein de certaines commissions spécialisées le conduisirent à élaborer une pensée réformatrice. Ensuite, et sur la base de ces éléments, nous mettrons au jour le processus d'élaboration des ordonnances en mettant l'accent sur les liens entre les enjeux politiques soulevés par ces nouvelles lois et la vision réformatrice de Pons et de ses hommes.

\section{Un arbitrista à la cour}

Voilà plus de vingt ans qu'au service de Dieu, du roi notre seigneur qui est au ciel, de Votre Majesté et de vos glorieux descendants ainsi que pour le bien temporel et spirituel des vassaux, j'essaie de déterminer par quels moyens la monarchie pourra durer encore des siècles (...) ;et après m’y être employé sur mes terres, à Rome et à Naples, je vins il y a plus de quinze ans à la cour avec don Juan de Zúñiga précepteur et premier majordome de Votre Majestéz

En novembre 1587, un an après la mort de Juan de Zúñiga y Requesens, Pons sollicita une audience 8 à don Juan de Idiáquez, ancien ambassadeur à Gênes puis à Venise, secrétaire d'État aux provinces du nord et à l'Italie depuis 1578 et l'un des plus proches collaborateurs du monarque. Par l'entremise d'Idiáquez, Pons va progressivement se faire connaître à la cour et, plus important encore, bénéficier de l'écoute et de la confiance des hommes de la Junta secreta $\underline{9}$. En œuvrant aux côtés de Juan de Idiáquez et de Cristóbal de Moura - tous deux membres de cette Junta -, Pons eut également à travailler avec d'autres hommes évoluant dans la sphère de la décision royale. De nombreuses lacunes documentaires empêchent d'établir avec précision la nature de ces relations. Cependant, certaines sources mettent en évidence la circulation de documents entre Pons et les secrétaires de Philippe II. Ainsi, de nombreuses propositions de l'arbitrista étaient examinées par les Conseils, les Juntas ou transmises au roi par l'intermédiaire de Mateo Vázquez, puis de Jerónimo Gassol10, ou encore de Juan de Ibarra, lequel occupait à cette époque le secrétariat du Conseil des Indes, celui de la Junta Grande et coordonnait les travaux de la Junta de Puerto Rico11. Ainsi, en novembre 1593, quelques semaines avant la publication des ordonnances, Jerónimo Gassol écrivait à Philippe II que « la raison pour laquelle nous n'avons pas étudié hier soir les courriers de Ibarra et de Pons fut l'absence de don Cristóbal [de Moura] ainsi que celle du prince notre seigneur et du marquis [de Velada] et l'affaire étant si importante, il m'a semblé de rigueur que tous [ceux de la Junta secreta] soient présents $\gg \underline{12}$.

Les papiers que nous avons pu dépouiller dans divers fonds d'archives montrent également que Pons était en contact avec des officiers des finances de divers horizons. L'un des principaux, Pedro 
De la consultation à l'ordonnance. Gaspar de Pons et la réforme des instituti...

Luis Torregrossa, veinticuatro de Séville, avait été nommé en 1580 premier Official del libro de caxa enquadernado para assentar la quenta y raz[ó]n de todas las rentas de la hazienda real, dont la mission était de tenir « les comptes généraux et particuliers de tout le trésor, tant de nos alcavalas et tercias 13 que des services et toute autre rente ordinaire et extraordinaire de la couronne des royaumes de Castille ainsi que de tout ce qui nous parvient des Indes »14. Torregrossa, dont les fonctions se résumaient à mettre au jour les fraudes tant à Séville qu'à Madrid, s'était confronté aux réseaux de corruption et aux conflits générés par les intérêts personnels des officiers royaux à tous les étages de l'appareil gouvernemental. En 1592, Pablo de Laguna, nommé gouverneur par intérim du Consejo de Hacienda15avait relancé la campagne de maîtrise des comptes de la couronne en nommant Torregrossa inspecteur des finances (contador)16. C'est à ce moment-là qu'on le voit entrer en contact avec Pons et participer avec ce dernier à plusieurs commissions d'experts. Il en fut de même avec le factor de la Casa de Contratación, Francisco Duarte, lequel faisait partie de l'équipe dirigeante de l'institution sévillane avec Pedro Gutiérrez Florez (président), Ochoa de Urquiza (contador) et Francisco Tello de Guzmán (trésorier) 17. Duarte était à cette époque l'un des principaux contacts de Pons en Andalousie. Pour finir, les liens de ce dernier avec d'autres arbitristas lui avaient permis d'acquérir la langue et les pratiques de l'arbitrismo $\underline{18}$.

Parallèlement aux réseaux dans lesquels Pons s'inséra, le rapport entre l'arbitrismo et l'usage de Juntas mérite également d'être approfondi19. L'inflation du nombre de Juntas dans les vingt-cinq dernières années du règne de Philippe II avait stimulé la venue à la cour, ainsi que la participation à la vie politique, de personnages nouveaux, désireux d'offrir leurs services au monarque. Ni ministres, ni conseillers, ces hommes venaient prendre part aux débats en cours en fonction de leur degré d'expertise, d'une expérience particulière ou bien encore parce qu'ils bénéficiaient de la bienveillance d'un président de Conseil. S'ils étaient nombreux parmi les officiers royaux de second plan à être conviés à ces réunions extraordinaires, certains - comme Pons - ne possédaient d'autres lettres de créance que d'avoir été au service d'un puissant.

L'usage de Juntas apparaît absolument central dans l'évolution de la mentalité et des pratiques politiques de l'époque car les Juntas conduisaient à concevoir l'action gouvernementale comme la réunion ponctuelle de spécialistes, en marge des travaux des grands Conseils, devant instruire une affaire déterminée. Ces organes véhiculèrent l'idée qu'aux côtés du système conciliaire d'autres espaces existaient où l'action politique était possible, peut-être libérée de cadres trop statiques et de conseillers jaloux de leurs prérogatives. Il y avait donc dans les Juntas un moyen idéal pour ceux que l'on nommait arbitristas d'accroître leurs chances d'être écoutés en venant à la cour.

Les principales idées de Pons sont consignées dans un document qu'il adressa aux membres de la Junta secreta en 1590 sous la forme d'une série de propositions numérotées de A à Z et se divisant en trois grandes sections 20 . La première s'attachait au désendettement du patrimoine royal et nobiliaire au moyen de solutions fiscales et monétaires, notamment en stimulant la frappe de monnaie. La seconde avait trait aux économies que l'on pouvait faire en réduisant certains frais somptuaires. Enfin, la troisième montrait comment, en appliquant les medios précédents et d'autres encore, on augmenterait les rentes de la couronne d'au moins trois millions de ducats par an. Au cours des années qui suivirent ses idées s'affinèrent et connurent certaines variations mais on peut dire que les principales lignes de politique économique de Pons étaient déjà définies.

Pour chacun de ces arbitrios, la Junta secreta décida, dans un bref commentaire, des institutions ou des personnages auxquels il devait être renvoyé. Le problème de la frappe de monnaie serait ainsi soumis à une Junta exceptionnelle que l'on allait créer à cette fin (il s'agissait pour l'essentiel 
de circonscrire à Ségovie la fabrication d'espèces sonnantes et trébuchantes et d'encourager la frappe de pièces plus faibles dans le but de « dévaluer la valeur des choses ») ; la proposition de vendre certaines juridictions de la couronne devait être remise au Consejo de Hacienda et celles des particuliers à la Cámara de Castilla ; quant à la dernière section, il revenait à Rodrigo Vázquez, alors président du Consejo de Hacienda, de l'examiner dans son ensemble « lors de la Junta qui se tient chez lui »21. De cette façon, les idées de Pons, à l'instar de celles d'autres personnages, commencèrent à circuler de main en main.

Un an plus tard, lorsque Philippe II instaura la Junta Grande22, Gaspar de Pons figura très vite parmi les hommes dont la participation fut la plus récurrente aux côtés de présidents de grands Conseils, de secrétaires, de conseillers. Mieux encore : les personnages tels que Pons n'étaient pas là pour écouter ou pour s'informer mais pour prendre la parole et soumettre leurs idées afin de contribuer à la résolution des problèmes financiers de la couronne. Toutefois, une certaine distance semble rapidement se creuser entre les propositions à court terme, largement préférées par les membres du Consejo de Hacienda $\underline{23}$, et les visions à long terme défendues, entre autres, par les arbitristas24. Au-delà de cet écart, il y avait aussi, chez Pons, une propension à proposer des solutions réformatrices ou supposant un renouvellement législatif. Aussi, Pons parlait-il de reformación concernant les bijoux, les laquais et le personnel de maison, ou encore évoquait-il la possibilité de plafonner les honoraires des avocats et des médecins $\underline{25}$.

Bien que les propositions de Pons aient été examinées presque quotidiennement entre février et juillet 1591, peu d'entre elles furent mises en ouvre et les débats durent être houleux en commission à en croire le ressentiment de l'arbitrista : «ce que je dis est précisément tout le contraire, à savoir que ce dont il s'agit est de trouver par quels moyens tous les vassaux de Votre Majesté pourront vivre riches et heureux et cesseront d'être opprimés par les élucubrations de ministres peu zélés et les solutions créées de toutes pièces par différents particuliers qui ne cherchent par là qu'à gagner leur pain » $\underline{26}$. La position de Pons était inédite : ne soutenant ni les conseillers, ni les arbitristas, il condamnait les nouveautés imaginées par les premiers - nouveautés auxquelles conduisait la recherche effrénée de nouveaux expédients financiers - et s'opposait aux recettes miracles issues de l'opportunisme courtisan des seconds.

Mais ce qui marquait la plus grande différence entre Gaspar de Pons et le reste des membres de la Junta Grande, était la conviction que sans une refonte en profondeur des organes centraux des finances de la couronne il était vain de chercher des arbitrios. Il faut, écrivait Pons au roi, «faire en sorte que les ministres de justice rendent justice et qu'ils respectent et fassent respecter les lois existantes ainsi que celles qu'il convient aujourd'hui d'édicter afin de bannir vices et péchés et de permettre aux vassaux d'être suffisamment riches et heureux et afin d'accroître les rentes royales pour que le patrimoine de Votre Majesté soit administré et dépensé comme il vous plaira »27. Le problème n'était pas nouveau et ce type d'argumentaire avait fini par devenir courant dans la littérature politique de l'époque. La spécificité de Pons fut d'aller jusqu'au bout du raisonnement et de proposer concrètement une réorganisation des organes financiers.

La vraie question, selon Pons, était de s'attaquer à un système gangréné au sein duquel s'étaient installées des habitudes qu'il fallait absolument proscrire. La « cause générale » venait selon lui de « la nature humaine »: « ceux qui possèdent des offices publics traitent davantage de leurs affaires personnelles que du bien public duquel ils sont peu à prendre soin comme il se doit »28. Par la suite, Pons précisait sa pensée en expliquant que, depuis près d'un siècle, il était devenu habituel que «les conseillers de gouvernement soient tous des juristes et que les mêmes personnes soient 
De la consultation à l'ordonnance. Gaspar de Pons et la réforme des instituti...

à la fois conseillers, juges de parties et juges d'inspection (visitas, residencias) »29. En dépit de ces accusations de fraude à peine voilées, Pons ne pensait pas que la substitution systématique des officiers et des ministres fût une solution viable. Il préconisait au contraire de "rénover et réorganiser les choses au moyen d'une Junta de personnes instruites, désintéressées et non employées [au service du roi] » $\underline{30}$, c'est-à-dire qui ne seraient pas susceptibles de défendre leurs intérêts - individuels ou collectifs - lors des débats.

Parti de cet ensemble de considérations, Gaspar de Pons en venait donc à réfléchir à l'amélioration structurelle et organisationnelle des institutions financières. Si le problème fondamental était que Conseils et ministres traitaient « d'affaires incompatibles » $\underline{31}$, la solution était naturellement de tendre vers une meilleure répartition des tâches. Aussi, écrivait-il, tout domaine politique doit être divisé en trois catégories d'officiers : «Les premiers, les conseillers, qui se chargent du gouvernement sans pouvoir dicter de sentence ni être juge d'inspection », "les seconds, les juges, auxquels il ne revient que la conduite des procès entre parties » et les derniers "nommés inspecteurs des finances (vehedores), chargés des visites d'inspection des ministres sans qu'ils ne puissent jouir d'aucun autre office public $» \underline{2}$.

L'office était ainsi au cœur des problèmes soulevés par Pons. Pour lui, les problèmes financiers de la couronne n'étaient pas seulement liés aux spéculations des négociants sévillans, à la fluctuation des taux d'intérêt ou encore aux fraudes en tous genres mais aussi au manque de diligence des agents du pouvoir et aux déficiences d'un appareil gouvernemental incapable de faire appliquer la volonté royale. Le projet de réforme de Pons s'attaquait donc directement à tous ceux qui, au sein des grands Conseils et des Juntas, occultaient leurs intérêts particuliers ou leurs incapacités derrière leur acharnement à trouver des expédients fiscaux. Dès lors, la question n'était plus de savoir où trouver des fonds mais comment cesser d'en perdre. De l'expertise financière au réagencement des rouages administratifs, la posture de Pons était passée de celle de fiscaliste à celle de réformateur.

\section{Le processus législatif}

Imaginer et concevoir [ces réformes] est une chose; les mettre en œuvre en est une autre $\underline{33}$

Le processus de rédaction de ces ordonnances n'a été que très peu étudié. Les spécialistes qui s’y sont penchés ont essentiellement voulu montrer le rôle qu'elles jouèrent dans l'affermissement du pouvoir des juristes (letrados) sur ce secteur du gouvernement34. Même s'il est vrai que les juristes continuèrent d'être largement représentés au sein des institutions financières, cette interprétation mérite d'être nuancée. En outre, l'enjeu des ordonnances n'était pas là. Pour ce qui nous intéresse, l'objectif de retracer ce processus législatif est double. D'une part, il s'agit de préciser certains aspects de la réforme qui n'ont pas encore été étudiés; et, d'autre part, nous voudrions montrer dans quelle mesure et par quels moyens les hommes qui eurent à leur charge de la préparer parvinrent ou non à l'infléchir selon leurs propres points de vue.

\section{La phase préliminaire}

En février 1592, Gaspar de Pons fit parvenir à la Junta secreta trois documents dont les deux principaux étaient de la teneur suivante : « dans le premier, Pons évoque l'entretien que nous, les trois de la Junta [secreta], devrions avoir avec lui afin de traiter des réformes; dans le second, il explique les raisons pour lesquelles ces réformes sont nécessaires et les bénéfices que l'on en obtiendra »35. Les hommes de la Junta semblaient enthousiastes et enclins à donner à Pons les 
moyens dont il aurait besoin pour leur élaboration. Comme lui, ils pensaient qu'il était inutile de multiplier les pragmatiques et autres décrets si par la suite ils n'étaient pas appliqués par les agents du pouvoir. Mais ils songeaient aussi que, même si Pons avait déjà posé de bonnes bases pour la réforme, de nombreux points devaient encore être traités afin que « son introduction soit mieux reçue $\triangleq \underline{36}$.

La réunion de Pons et des hommes de la Junta secreta eut lieu à la fin mai. Toutefois, si la Junta soutenait pleinement l'entreprise de Pons, il semblerait que le roi ait été quelque peu circonspect, dans un premier temps du moins. Aussi, Philippe II commanda-t-il que Pons commence par en informer, «individuellement et en grand secret », Pedro Barbosa, don Luis de Castilla, Fadrique Furió Ceriol et don Juan de Ancora. Quant à ces derniers, ordonnaient les membres de la Junta secreta, "qu'ils l'entendent et renvoient leurs opinions par lettre scellée à l'attention de Sa Majesté via le secrétaire Gassol (...) »37. Il s'agissait pour l'heure de savoir si une réforme était envisageable. Il est particulièrement intéressant d'observer que, sans doute sur les conseils de Pons, le projet du monarque de consulter préalablement certains personnages n'incluait aucun membre de la haute administration financière. Avant de soumettre le projet à une commission et avant que ne s'ouvrent les débats, Philippe II semblait vouloir rassembler les opinions de personnes ne possédant pas d'office dans ce domaine politique ou, du moins, pas au niveau de l'administration centrale. En sollicitant un contrôleur des finances résidant à Séville, un arbitrista, un penseur politique et un membre de la noblesse on collecterait les idées et préconisations d'observateurs évoluant dans des sphères du politique tout à fait distinctes et procédant de formations et de carrières notablement opposées $\underline{38}$.

Un an plus tard, en février 1593, sur recommandation de la Junta secreta, Pons demanda au roi à être entendu par une Junta extraordinaire qui aurait pour mission d'examiner ses propositions. Après avoir présenté en quelques lignes les raisons pour lesquelles il souhaitait désormais avoir affaire à une commission réformatrice, il indiquait les personnes qui, selon lui, possédaient l'expérience et les connaissances nécessaires afin d'entamer une réflexion de fond sur le sujet. Les raisons de ses choix s'avèrent extrêmement éloquentes : «Je propose des théologiens, des juristes, des gentilshommes et des officiers des finances car il est certain que, dans la confection de lois et dans la mise en ordre de l'administration de la justice et des finances de Votre Majesté, il est nécessaire de réunir des personnes issues de toutes ces professions $\gg \underline{39}$. Cette approche pluridisciplinaire de la fabrique des lois, fondée sur les pratiques de la Junta Grande, excluait une nouvelle fois conseillers et présidents de Conseils en même temps qu'elle incitait à une forme participative de gouvernement. De toute évidence, il s'agissait de mettre à l'écart des débats ceux que les ordonnances pouvaient menacer tout en permettant à différents acteurs, qu'ils soient officiers de l'inspection des finances ou spécialistes de questions militaires, diplomatiques ou encore religieuses, de pouvoir dialoguer afin de donner corps à la réforme la plus efficace possible.

Quelques semaines plus tard, sur la base des propositions de cette Junta spécifique, la Junta secreta présenta au roi le brouillon d'un texte législatif qui allait devenir l'ordonnance du 20 novembre $1593 \underline{40}$

\section{La réforme}

La Junta secreta avait préparé quatre documents. Le premier, intitulé « Resoluci[ó]n de su Md. para la compostura de los tribunales de hazienda »41, s'attachait à dénombrer les membres devant intégrer chaque institution ainsi que leurs fonctions. Les trois suivants décrivaient les compétences 
De la consultation à l'ordonnance. Gaspar de Pons et la réforme des instituti...

et les aspects fonctionnels de chacun des tribunaux - le Consejo de Hacienda, la Contaduría Mayor de Hacienda et la Contaduría Mayor de Cuentas $\underline{42}$. Finalement, plusieurs feuillets de la main de Philippe II revenaient sur quelques aspects généraux et amendements formels $\underline{43}$. Dans les grandes lignes, il s'agissait de : 1/ attribuer à chaque instance un champ de compétences resserré et 2/ définir avec précision les fonctions et les pratiques des divers officiers. Afin de ne pas trop alourdir la démonstration nous nous en tiendrons à présenter les dispositions qui serviront le mieux notre propos.

D'abord, il y a le fait que le Consejo de Hacienda, auquel le brouillon de la Junta secreta attribuait encore une fonction judiciaire sur les domaines financiers lui incombant, en fut totalement privé par la main du roi : « les litiges [ne doivent pas être remis au Consejo de Hacienda] même s'ils ont trait aux affaires à sa charge $» \underline{4} 4$. Une telle résolution était inédite et émanait sans aucun doute possible de Pons. Mais si les hommes de la Junta secreta n'en avaient pas saisi les réelles implications, Philippe II, en revanche, avait perçu la portée de cette mesure sur laquelle il insista plusieurs fois dans les documents de travail de la Junta secreta puis dans la version finale des ordonnances: "qu'en Conseil ne soit examiné aucun litige entre parties en rapport aux rentes ordinaires, extraordinaires ni ayant trait à aucun expédient et que l'on n'en ait aucune connaissance ; que tous [les litiges] soient remis et traités au sein de la Contaduría Mayor de Hacienda »45. D'autre part, la Junta secreta préconisait un renvoi systématique au Conseil de toutes les consultations en provenance des diverses Juntas, dont la Junta Grande et sa cohorte de commissions constituaient implicitement le modèle.

De façon assez semblable, la Contaduría Mayor de Hacienda serait désormais scindée en deux chambres : le tribunal des inspecteurs des comptes (contadores), en charge des negocios del libro y administración de la hacienda - autrement dit : des fraudes administratives - et le tribunal des oidores devant étudier les procès et litiges entre les parties. A l'instar de l'agencement global des trois instances, l'organisation interne de la Contaduría était également soumise à une structuration par cloisonnement des tâches faisant écho aux conseils de Pons. En résumé, écrivait Philippe II en marge du brouillon : "la Contaduría Mayor de Hacienda conserve à sa charge tout ce qui lui incombait jusqu'à ce jour, à quoi s'ajoute l'instruction des procès (pleitos) qui étaient à la charge du Consejo »46. Pour sa part, la Contaduría Mayor de Cuentas ne reçut pas de modification substantielle et allait continuer d'agir de la même façon que par le passé, c'est-à-dire comme un tribunal des comptes généraux $\underline{4}$.

L'ensemble de ces salles, y compris celles de la Contaduría Mayor de Cuentas, agissait sous la supervision du président du Consejo, lequel avait autorité pour assister à n'importe laquelle de ces réunions autant de fois et aussi régulièrement qu'il le jugeait opportun $\underline{48}$.

Ces premières considérations sont fondamentales en ce qu'elles entérinent la volonté du monarque - encouragée par les travaux des hommes de Pons - de répartir entre les deux principales instances financières - le Consejo et la Contaduría Mayor de Hacienda - l'exercice de la justice de l'exercice du gouvernement. En devenant à la fois le réceptacle des travaux des Juntas, l'administrateur des rentes liées au patrimoine royal et libéré de ses fonctions judiciaires, le Consejo de Hacienda apparaissait désormais comme l'institution en charge d'élaborer et de conduire la politique économique et financière de la couronne. Mais cette apparente scission des fonctions judiciaires et politiques - que les intitulés donnés aux instances (Consejo Real de Hacienda et Tribunal de los contadores de la Contaduría Mayor de Hacienda) confirment pleinement - était loin de signifier un cloisonnement absolu. De fait, les ordonnances spécifiaient que le Consejo de Hacienda devait 
être composé d' "un président, deux membres du Conseil royal, deux des quatre contadores de la Contaduría mayor de hacienda (...) et quelques autres personnes que [le roi] nommerai[t] si [il] le jugeai[t] opportun $» \underline{49}$. La présence des principaux inspecteurs des finances de la Contaduría au sein du Consejo met en évidence la nécessité d'articuler l'activité de l'une et l'autre institution et de favoriser la circulation d'informations afin de donner au Consejo les moyens d'instruire les affaires et de donner à ses arrêts une plus grande cohérence.

De façon similaire, la séparation pure et simple en deux salles de la Contaduría Mayor de Hacienda semblait poser quelques problèmes pratiques. C'est pourquoi certains officiers devaient être employés à faire le lien entre elles. A cette fin, la Junta secreta préconisait l'intervention organisée et délimitée dans un cadre précis de certains officiers dans des réunions transversales : " que le contador et l'oidor les plus anciens se réunissent avec le procureur une à deux fois par semaine ainsi qu'avec l'officier de plume attaché aux rentes et les contadores del libro afin de prévenir et de traiter certaines choses ayant trait à l'administration des finances $» \underline{50}$. Cette sous-commission de la Contaduría avait à sa charge de traquer les dysfonctionnements, les impayés et les diverses difficultés rencontrées - ou provoquées - par les agents du gouvernement. Par la suite, leurs résolutions devaient être transmises au tribunal de la Contaduría et soumises à l'approbation de la réunion plénière de l'institution.

Concernant les documents traitant spécifiquement des deux Contadurías, un autre problème s'ajoutait. Les alinéas réservés à la définition de leurs compétences en tant que collèges étaient largement réduits au profit d'une explicitation des tâches des officiers. La redéfinition des offices le nombre des officiers de chaque chambre et leurs fonctions, les documents qu'ils étaient autorisés à signer, ou pas, et sous quelles conditions, etc. - faisait l'objet de descriptions précises, de même que les procédures de réunion (horaires, ordre des votes).

Par exemple, les ordonnances spécifiaient que les officiers des livres de comptes - qui en étaient les propriétaires - ne devaient plus rien y consigner qui n’ait été préalablement ordonné par le tribunal de la Contaduría mayor de hacienda. Il leur était également exigé de dater et signer systématiquement tous les paiements effectués. De même, le brouillon indiquait - ce que la version finale des ordonnances ne stipulait pas - qu'il était catégoriquement interdit à ces officiers de montrer les livres de comptes aux négociants et que seuls les ministres du roi y étaient autorisés sous réserve d'obtenir préalablement l'accord du président du Consejo $\underline{51}$.

Pour leur part, les contadores de la Contaduría Mayor de Cuentas ne pouvaient désormais plus jouir d'autres offices royaux, ce qui avait vraisemblablement été le cas jusqu'ici et qui faisait partie, rappelons-le, des points centraux de l'argumentation de Pons. Ils devaient aussi à présent entendre de vive voix les officiers ayant produit des rapports inexacts ou au sujet desquels quelque doute demeurait, la mise par écrit de leurs résultats par lesdits contadores n'étant plus suffisante. Enfin, ordre était donné afin que deux contadores de la Contaduría Mayor de Cuentas se chargent le plus rapidement possible de la mise à jour des comptes (en particulier pour ce qui concernait les retards de paiements des militaires) $\underline{52}$.

Finalement, les nominations aux offices des finances devaient désormais suivre une procédure précise. Dans un premier temps, les deux Contadurías devaient s'informer des hommes les plus à même de conduire les tâches à pourvoir. Il leur revenait ensuite de faire remonter leurs listes de prétendants au Consejo lequel était en charge de consulter le roi et de recevoir de lui ses consignes $\underline{53}$. 
De la consultation à l'ordonnance. Gaspar de Pons et la réforme des instituti...

Le processus d'élaboration des ordonnances - circulation de billets, de documents, de brouillons, échanges épistolaires divers - dut être intense pendant l'été 1593 ainsi qu'en témoignent ces quelques lignes des hommes de la Junta secreta : "Tous les papiers contenant les résolutions et les notes marginales que Votre Majesté y fait figurer ont été réexaminés en Junta [secreta] (...) ; et comme il semble plus opportun que tout soit noté sur un seul document, nous avons rassemblé la substance de ce qui était écrit en marge de tous les papiers afin que l'on puisse continuer consécutivement à annoter ce qui devra l'être $(. .). » \underline{54}$.

Aussi ce ne fut sans doute pas par hasard que, début novembre, le roi demanda à Jerónimo Gassol si les ordonnances devaient être rédigées à la première personne du pluriel ou du singulier. Le secrétaire, auquel on avait commandé d'aller vérifier dans les pratiques antérieures, notamment parmi les ordonnances des rois catholiques, répondit que: «pour ce qui est des lois, des ordonnances et des pragmatiques, qui sont choses perpétuelles et que les rois successifs doivent faire respecter du moment qu'ils ne les révoquent pas, il a presque toujours été d'usage d'employer le Nous - en particulier en disant «nous ordonnons »- non seulement parce que ces choses sont traitées par plusieurs personnes, mais aussi au nom des successeurs qui doivent en ordonner l'application $» \underline{55}$.

Le « Nous » royal s'était donc substantiellement modifié pour devenir un «pluriel collaboratif » avant d'être l'expression de la souveraineté. La formulation contenue dans les ordonnances publiées quelques semaines plus tard, quoique plus ambiguë ne trahissait pas ce changement et mettait à l'honneur les pratiques qui avaient permis l'édiction de la nouvelle loi : "ayant été examiné et débattu ce qu'il conviendrait de déterminer et d'ordonner à ce sujet, et Nous ayant consulté, il a été décidé que nous devions ordonner ce qui suit $\gg \underline{56}$.

\section{Conclusion}

À s'en tenir à la lettre des ordonnances, à restreindre l'approche historienne de la fabrique des lois à l'expression de la volonté royale, on ne peut percevoir ce que l'étude attentive des coulisses du gouvernement permet de mettre en lumière : la complexité d'un processus dans sa dimension politique et dans sa mise en œuvre gouvernementale. Cette réforme en profondeur des organes financiers de la monarchie ne saurait donc être tenue pour être la simple démarche du monarque ni d'ailleurs pour une habile manœuvre des juristes afin de se maintenir au pouvoir - mais doit avant tout être entendue comme le résultat de travaux collectifs, de semaines de débats préliminaires, de consultations, de séances de délibération ayant réuni de multiples personnages. Aussi, bien plus que le texte final des ordonnances, c'est le cheminement intellectuel de Pons ainsi que son acquisition des pratiques de gouvernement, depuis son arrivée à la cour jusqu'à l'élaboration de la réforme, qui doivent être utilisés comme les fils conducteurs de cette séquence historique : celle de la lente gestation d'un projet de renouvellement législatif. De sorte que cette étude nous renseigne non seulement sur le contexte politique et les enjeux qu'une telle réforme induisait pour le système conciliaire mais encore constitue-t-elle un angle d'approche particulièrement fécond afin de mettre au jour les pratiques de gouvernement. Car, on l'a vu, l'usage de commissions spécifiques, les consultations ponctuelles d'experts et l'élaboration de documents de travail apparaissent ici comme indissociables du processus législatif. Or, il s'avère que, bien plus qu'une toile de fond, cet ensemble de pratiques se trouvait au cœur des enjeux politiques gravitant autour des ordonnances.

Ces pratiques, fondées sur le besoin de réunir des opinions diverses, constituaient une grande opportunité pour ceux qui, à la cour, souhaitaient étendre leur champ d'influence. Il en résultait 
ainsi une reconfiguration du service du roi. Car la nécessité que l'on pouvait avoir de juristes (letrados) cessait de prévaloir sur d'autres domaines d'expertise. La collaboration entre ces derniers et, en particulier, les contadores des diverses chambres des Contadurías, stipulée dans les ordonnances, témoigne à la fois d'un enrichissement des outils de gouvernement et d'un décloisonnement de la structure sociopolitique. Au sein de l'administration centrale des finances, aussi bien que dans les Juntas ayant participé à sa réforme, l'opposition stricte entre juristes et noblesse de conseil n'était plus guère pertinente à la fin du règne de Philippe II. De fait, dans cette administration bien plus qu'ailleurs, les juristes étaient depuis longtemps devenus les principaux acteurs. La question pour nous n'est donc pas de déterminer si ces derniers parvinrent à se maintenir au pouvoir mais de saisir comment, et en quoi, le curseur se déplaça depuis la fracture sociale traditionnelle entre aristocrates et spécialistes du droit vers des clivages davantage liés à la technicité gouvernementale. De fait, les juristes eux-mêmes tendaient à cette époque à élargir leur expertise et à compléter leur connaissance de la loi par d'autres savoirs spécialisés. La complexité de la machine gouvernementale élaborée par Philippe II excédait largement la capacité des hommes de lois à la contrôler. Le droit tendait à devenir un outil de gouvernement parmi d'autres.

\section{Notes}

1 Ce document, conservé en : Biblioteca del Palacio Real (cité après BPR), III/3711, doc. 13, était signé par Rodrigo Vázquez, alors président du Conseil royal et ancien président du Consejo de Hacienda, et par trois autres membres de son Conseil. Comme c'était la procédure, la signature de Juan Vázquez, secrétaire de la Cámara de Castilla et du Conseil Royal apparaît en fin de document. Composées de onze folios et divisées en plusieurs sections, les ordonnances revenaient sur l'ensemble des attributions des instances de gouvernement et sur les fonctions des officiers des finances.

$\underline{2}$ BPR, III/3711, doc. 13, fol. 1r. Sauf mention contraire, c'est nous qui traduisons.

$\underline{3}$ Concernant les questions fiscales, nous renvoyons à : FORTEA PÉREZ J. I., Monarquía y Cortes en la Corona de Castilla : las ciudades ante la política fiscal de Felipe II, Valladolid, 1990 ; DUBET A., Réformer les finances au Siècle d'Or. Le projet Valle de la Cerda, Clermont-Ferrand, 2000 ; ID., Le servicio de los 8 millones (1588-1590) ou la négociation érigée en principe d'action dans Ibérica, $\mathrm{n}^{\circ}$ 11, 1999, p. 47-65. Voir également les travaux de MARCOS MARTín A., en particulier : España en los siglos XVI, XVII y XVIII : economía y sociedad, Barcelona, 2000. En termes plus généraux : CARANDE R., Carlos V y sus banqueros, Barcelona 2000 ; ID., Gobernantes y gobernados en la Hacienda de Castilla (1536-1556), dans Siete estudios de Historia de España, Barcelona, 1971 (voir notamment les pages 109 à 122) ; UlLOA M., La hacienda real de Castilla en el reinado de Felipe II, Madrid, 1977 ; Fernández Albaladejo P., Fragmentos de monarquía, Madrid, 1992.

4 Sur l'arbitrismo, voir : VILAR J., Literatura y economía : la figura satírica del arbitrista en el Siglo de Oro, Madrid, 1973 ; SÁncheZ Molledo J. M., Arbistristas aragoneses de los siglos XVI y XVII. Textos, Zaragoza, 2009 ; GUTIÉREZ NIETO, El pensamiento económico, político y social de los arbitristas, dans Historia de España, éd. Ramón Menéndez Pidal, t. XXV, El siglo del Quijote (1580-1680), Madrid, 1988, p. 235-355 ; DUBET A., L'arbitrisme : un concept d'historien ?, dans Cahiers du Centre de Recherches Historiques (EHESS), n² 24, avril 2000, pp. 141-167.

$\underline{5}$ L'historiographie du XIX ${ }^{\mathrm{e}}$ siècle et du premier $\mathrm{XX}^{\mathrm{e}}$ a largement relayé cette lecture de la décadence de la monarchie espagnole de laquelle les arbitristas s'étaient fait les principaux 
De la consultation à l'ordonnance. Gaspar de Pons et la réforme des instituti...

théoriciens. Certains spécialistes ont depuis révisé cette lecture des choses : RuIZ IBÁÑ̃Z J. et VINCENT B., Los siglos XVI-XVII : política y sociedad, Madrid, 2007.

6 Sur Gaspar de Pons : SemPere y GuARINos J., Biblioteca española económico-política, Madrid, en la imprenta de Sancha, 1801 ; Colmeiro M., Biblioteca de los economistas españoles. Siglos XVI, XVII, XVIII, Madrid, 1953-1954 ; VILAR J., Literatura y economía..., op., cit. ; ForTEA PÉREZ J. I., Entre dos servicios : la crisis de la hacienda real a fines del siglo XVI. Las alternativas fiscales de una opción política (1590-1601) dans Studia H. Ha Moderna, t. 17, 1997, 63-90 ; DUBET A., Réformer les finances..., op., cit. ; SIMÓN TARRES A., Construccions politiques i identitas nacionals. Catalunya i els origens de l'estat modern espanyol, Barcelona, 2005 ; SÁNCHEZ D. M., El deber de consejo en el estado moderno : las Juntas "ad hoc" en España (1474-1665), Madrid, 1993 ; ESPEJO C., Enumeración y atribuciones de algunas Juntas de la Administración española desde el siglo XVI hasta el año 1800, dans Revista de Biblioteca, Archivo y Museo, t. 8, N 32, 1931, p. 352-362.

Z Biblioteca Nacional de España (cité après BNE), ms. 8526. Billet de Gaspar de Pons à Philippe III, non daté. Juan de Zúñiga y Requesens, prince de Pietraprecia, était revenu de Naples à la fin de l'année 1585 sur ordre de Philippe II afin de superviser les affaires à Madrid pendant que le monarque effectuerait son voyage à Monzón. (Cf. de HERRERA A., Historia general del mundo de XVII años del tiempo del rey don Felipe II. De M.D.LIII hasta el de M.D.LXX, Madrid, Imprenta de Pedro Madrigal, 1601, t. II, Chapitre XV, fol. 443 et suivants).

8 VILAR J., Literatura y economía..., op ., cit., p. 178. L’historien mentionne un memorial qu'à cette occasion Pons aurait transmis à Juan de Idiáquez. Nous n'avons malheureusement pas retrouvé ledit document.

9 Au sujet de cette Junta, plus connue sous le nom de Junta de Noche ou Junta de Gobierno, voir les travaux de l'équipe de recherches de José Martínez Millán ainsi que Escudero J. A., Los secretarios de Estado et Felipe II (1474-1724), Madrid, 1969etID., Felipe II. El rey en el despacho, Madrid, 2002. Sur Idiáquez en particulier, voir l'étude assez riche, quoique historiographiquement un peu datée, de PÉREZ Mínguez F., Don Juan de Idiáquez, embajador y consejero de Felipe II, San Sebastián, 1934.

10 Mateo Vázquez fut le principal secrétaire de Philippe II à partir de la mort du cardinal Espinosa survenue en 1572. C'est lui qui fut à l'origine de la création de la Junta secreta dont il était le rouage principal. Jerónimo Gassol prendra la suite de Mateo Vázquez, son beau-frère, à la mort de celui-ci en mai 1591. Sur Mateo Vázquez, voir : LovetT A. W., Philip II and Mateo Vázquez de Leca : the government of Spain (1572-1592), Genève, 1977.

11 Sur la Junta Grande, voir note 22, infra. Bien peu de travaux mentionnent le cas de la Junta de Puerto Rico qui semble avoir été l'une des premières instances dont la finalité était l'administration de l'espace atlantique. Comme c'est bien souvent le cas, nous n'avons pas trouvé de documents émanant directement de cet organe. Seuls demeurent les courriers et billets de Juan de Ibarra en charge de synthétiser les débats et les comptes rendus de la Junta de Puerto Rico afin qu'ils soient examinés par la Junta secreta.

12 Archivo de la Biblioteca Zabálburu (cité après AZ), Carpeta (cité après C.) 145, doc. 178. Billet du secrétaire Jerónimo Gassol à Philippe II, du 10 novembre 1593. En outre, ce n'est sans doute pas par hasard que, hormis ses multiples fonctions au sein de l'administration centrale de la 
monarchie, Juan de Ibarra figure également parmi les arbitristas. Cf. CORREA CALDERÓN E., Registro de arbitristas, economistas y reformadores españoles (1500-1936), catálogo de impresos y manuscritos, Madrid, Fundación Universitaria española, 1981.

13 Alcabalas : taxes qui frappent toutes les transactions égales en théorie à $10 \%$ de la valeur du bien vendu, en pratique ramenées à $4 \%$ environ. Elle est payée par le vendeur ce qui tend à réduire la capacité d'entreprise. Tercias : grâce accordée par l'Église au pouvoir royal. Concession au roi de $2 / 9$ e de la valeur des dîmes. Les alcabalas et tercias sont abonnées ensemble et donc recouvrées en même temps. (Cf. CARANDE R., Carlos V..., op., cit., p. 227 et suivantes).

14 Archivo General de Simancas (cité après AGS), Escribanía Mayor de Rentas (cité après EMR), Quitaciones de Corte (cité après QUIT), legajo (cité après leg.) 38, fol. 644.

15 De Carlos Morales C. J., El consejo de hacienda de Castilla. Patronazgo y clientelismo en el gobierno de las finanzas reales durante el siglo XVI, Valladolid, 1996, p. 159 et suivantes.

16 AGS, EMR, QUIT, leg. 38, fol. 672.

17 Sur les relations entre ces personnages et les membres de l'administration centrale, voir les consultations du Conseil des Indes au cours des années 1594-1596 : Archivo General de Indias (cité après AGI), Indiferente (cité après Indif.), leg. 742-744. Voir également : ScHAFFER E., El Consejo Real y Supremo de las Indias, Séville, 1935.

18 Pons possédait « un immense passé d'arbitrista plus ou moins écouté et ses écrits constituent un exemple typique du style de pensée et du langage des arbitristas ». Cf. VILAR J., Literatura y economía..., op., cit., p. 178. Loin d'être homogènes les textes des arbitristas révèlent néanmoins des pratiques communes (élever au roi des propositions, insistance auprès des ministres à la cour pour être entendus) et des constantes discursives (soigner les maux de la monarchie, assurer le bien être et la richesse des royaumes en soulageant leur charge fiscale, etc.).

19 GUitÉRrez Nieto J. I., El pensamiento económico..., op., cit., p. 236.

20 AZ, C. 133, Grupo Documental (cité après GD.) 1, doc. 153.

$\underline{21}$ Ibid., fol. 4.

22 Cette Junta rassemblait un très grand nombre de spécialistes et se divisait en commissions spécifiques afin de statuer sur les divers expédients. Il n'existe à ce jour aucune monographie sur la Junta Grande. Quelques éléments toutefois sont consultables dans : DUBET A., Réformer les finances..., op. cit. ; de CARLos Morales C. J., El consejo de hacienda de Castilla..., op. cit. ; LovetT A. W., Philip II and Mateo Vázquez de Leca..., op., cit. ; BALTAR Rodríguez J. F., Las Juntas de gobierno en la Monarquía hispánica (siglos XVI-XVII), Madrid, 1998.

23 Philippe II insista beaucoup au cours de l'année 1591 sur la nécessité de trouver des expédients dont la mise en place et les résultats devaient être rapides. On peut citer à titre d'exemple l'emprunt (empréstito) à demi forcé défendu par Rodrigo Vázquez, président du Consejo de hacienda, et qui connaîtra un succès fluctuant au cours de la décennie.

24 C'est bien sûr le cas de Pons, mais aussi celui de Agustín Álvarez de Toledo qui conduisit une commission en faveur d'un impôt sur la farine qui devait se superposer au service des Millones ou 
De la consultation à l'ordonnance. Gaspar de Pons et la réforme des instituti...

encore celui de Luis Valle de la Cerda lequel envisageait la création d'erarios públicos. Cf. DuBET A., Réformer les finances..., op., cit. ; UlloA M., La hacienda real de Castilla..., op., cit.

25 Sur toutes ces propositions, voir en particulier : Instituto Valencia de Don Juan (cité après IVDJ), Expediente (cité après E.) 43, Caja (cité après C.) 55, doc. 169, n.f. Junta secreta du 10 mai 1591 ; IVDJ, E. 43, C. 55, doc. 170, n.f. Junta secreta du 13 mai 1591 ; IVDJ, E. 43, C. 55, doc. 177, n.f. Junta secreta du 24 mai 1591 ; IVDJ, E. 43, C. 55, doc. 180, n.f. Junta secreta du 4 juin 1591.

$\underline{26}$ AZ, C. 133, doc. 155, fol. 1.

27 IVDJ, E. 43, C. 56, doc. 386 bis, n.f.

28 BNE, VE. 28, n²6, fol. 273.

29 Ibid., fol. 273.

$\underline{30}$ Ibid., fol. 274. La notion de désintérêt revenait fréquemment dans les textes de Pons. Ne jouir d'aucun office royal en était la garantie. Selon Pons, il était devenu nécessaire que des hommes extérieurs à l'administration participent au gouvernement. Une façon bien sûr de légitimer et de promouvoir sa propre position.

31 Ibid., fol. 273.

32 Ibid., fol. 278.

$\underline{33}$ AZ, C. 133, GD. 1, doc. 154, fol. 1. Billet de Jérónimo Gassol à Philippe II, non daté.

34 C. J. DE CARLOS MORALES, op., cit., p. 162-164 ; Fernández Albaladejo P., Fragmentos de monarquía..., op. cit., p. 123-124.

35 IVDJ, E. 43, C. 55, doc. 268, n.f. Junta secreta du 13 février 1592.

$\underline{36}$ Ibid.

37 Ibid. Pedro Barbosa était une courroie de transmission du pouvoir à Séville. En tant que proveedor general, de multiples missions lui furent confiées visant au contrôle des marchandises en provenance des Indes (Cf. AGS, Consejo y Juntas de Hacienda (cité après CJH), leg. 330, carpeta (cité après carp.) 14 et leg. 342, carp. 16 ; AGS, Guerra y Marina (cité après GYM), leg. 411, doc. 111 ; AZ, C. 134, doc 125 et C. 145, doc. 39. Sur son rôle de proveedor general : AGI, Indif., leg. 743, fo 119). Fadrique Furió Ceriol était un célèbre humaniste et penseur politique valencien de la seconde moitié du $\mathrm{XVI}^{\mathrm{e}}$ siècle et l'auteur, entre autres, de El concejo y consejeros del príncipe (1559) (Cf. DíAz DíAz G., Hombres y documentos de la filosofía española, Madrid, 1988). Juan Antonio de Ancora faisait partie des arbitristas spécialistes de l'exploitation minière (Cf. Gimeno E. B. et Royo J. A. M., La minería aragonesa en la cordillera ibérica durante los siglos XVI y XVII : evolución económica, control político y conflicto social, Saragosse, 2013, p. 41).

$\underline{38}$ Malheureusement, nous n'avons pas retrouvé dans la documentation les réponses de chacun de ces personnages qui auraient permis d'en savoir plus sur le positionnement de certains secteurs politiques ou sur celui d'un homme aussi aguerri que Furió Ceriol (nous ne savons même pas si ce dernier y répondit, sa mort étant survenue ce même mois de mai 1592). Sur les longs mois qui séparent cette première phase de la suite, la documentation se tait et les quelques 
éléments glanés ça et là ne permettent nullement de retracer avec certitude le fil de l'histoire.

39 IVDJ, E. 43, C. 56, doc. 386 bis, n.f. Une fois encore on ne peut que constater la variété des personnages réunis autour de Pons. Aux côtés de Pedro Barbosa et de Pedro Luis Torregrossa dont nous avons déjà parlé (Cf. respectivement notes 37 et 16, supra), apparaissent, dans la liste de Pons, Bernardino de Mendoza, l'illustre ambassadeur espagnol en Angleterre et en France dans les années 1570-80; don Juan de Acuña Vela, capitaine général d'artillerie qui deviendra membre du Conseil de Guerre en 1595 (Cf. Martínez Millán J., Felipe II : la configuración de la monarquía hispana (1527-1598), Valladolid, 1998) ; Pedro Franqueza, à cette époque secrétaire du royaume de Valencia au Conseil d'Aragon, et qui deviendra l'un des favoris du duc de Lerma ; le doctor Francisco Terrones del Caño, célèbre prédicateur, devenu évêque de Tuy en 1601 (Cf. BELTRÁN HEREDIA V., Miscelánea Beltrán de Heredia : colección de artículos sobre historia de la teología española, vol. 1, Salamanque, 1972, p. 284.) ; le doctor don Melchor de Soria y Vera, spécialiste de la production agraire et futur évêque de Troya ; don Beltrán de Guevara - il s'agit probablement du troisième du nom - qui effectua notamment une visite d'inspection à Naples à l'époque où Pons s'y trouvait encore ; et, pour finir, le père Luis de Mendoza et don Francisco de Fonseca.

40 AZ, C. 182, doc. 39-46.

41 AZ, C. 182, doc. 40.

42 AZ, C. 182, doc. 41, 42 et 43. Cf. UllOA M., La hacienda real de Castilla..., op., cit.

43 AZ, C. 182 , doc. 46.

44 AZ, C. 182, doc. 41, fol. 1. Sur les domaines d'intervention du Conseil, et à l'exception des rentes extraordinaires que le monarque attribua à la Contaduría Mayor de Hacienda, un consensus avait été adopté sur la hacienda por mayor, les asientos, les arbitrios et les rentes liées à la vente des offices.

$4 \underline{5}$ BPR, III/3711, doc. 13, fol. 3r.

46 AZ, C. 182, doc. 42 , fol. 2.

47 CARAnde R., Carlos V..., op., cit., p. 197 ; Ulloa M., La hacienda real de Castilla..., op., cit., p. 60 et suivantes.

48 BPR, III/3711, fol. 2r.

49 Ibid., fol. $1 \mathrm{v}$.

50 AZ, C. 182, doc. 42, fol. 1.

51 Ibid., fol. 1-2.

52 AZ, C. 182, doc. 43, fol. 1-2.

53 AZ, C. 182, doc. 46, fol. 3.

54 IVDJ, E. 43, C. 56, doc. 418, n.f. Junta secreta du 27 juillet 1593. Une autre commission, composée du fiscal Ruypérez, de Pablo de Laguna (actuel gouverneur des finances par interim) et 
De la consultation à l'ordonnance. Gaspar de Pons et la réforme des instituti...

de Rodrigo Vázquez (ancien président du Consejo de hacienda qui avait été promu à la présidence du Conseil royal en 1592), fut chargée à la fin août de la mise au propre des divers papiers (Cf. BNE, ms. 18633/63 et C. J. DE CARLOS MORALES, op., cit., p. 163).

55 AZ, C. 145, doc. 175, n.f. Billet de Jerónimo Gassol à Philippe II, daté du 2 novembre 1593. C'est nous qui soulignons.

$\underline{56}$ BPR, III/3711, doc. 13, fol. 1. C'est nous qui soulignons.

PDF généré automatiquement le 2020-07-14 00:41:22

Url de l'article : https://popups.uliege.be:443/1370-2262/index.php?id=421 